\title{
NUMERICAL AND EXPERIMENTAL STUDY OF ASR IN CONCRETE AT THE MESO-LEVEL
}

\section{JOAQUÍN LIAUDAT, CARLOS M. LÓPEZ AND IGNACIO CAROL}

\author{
School of Civil Engineering-Barcelona (ETSECCPB) \\ Technical University of Catalonia (UPC), \\ E-08034 Barcelona \\ e-mail: joaquin.liaudat@upc.edu,carlos.maria.lopez@upc.edu, ignacio.carol@upc.edu
}

Key words: ASR, Durability Mechanics, Numerical Modeling

\begin{abstract}
In this paper, an ongoing research project on the mechanisms of concrete deterioration due to Alkali-Silica Reaction (ASR) is presented, together with some preliminary results. The research program includes both experimental and numerical modelling work.

The first part includes the development of two new experimental setups, one devoted to study the ASR expansion mechanisms at the level of a single matrix-aggregate interface and the other to the study of ASR expansions of cubic concrete specimens under triaxial confinement.

The second part includes the formulation and numerical implementation of a chemo-mechanical model for ASR expansions in concrete at the meso-level. The model considers three main diffusion/reaction field equations for the concentrations of silicates, calcium and alkalis in concrete pore solution, complemented by a number of chemical kinetics and chemical equilibrium equations. The volume fraction distribution of the solid constituents of the hardened cement paste and the reaction products evolve with the progress of the reaction, determining the diffusivity properties of the material and, eventually, inducing expansions.
\end{abstract}

\section{INTRODUCTION}

Despite concrete cracking and swelling due to ASR has been studied since it was first reported by Stanton in 1940 [1], the involved mechanisms are not yet completely understood.

In this paper, an ongoing research project on the mechanisms of concrete deterioration due to ASR is presented, together with some preliminary results. The research program includes both experimental and numerical modelling work.

\section{EXPERIMENTAL WORK}

The first part of the research project includes the development of two new experimental setups, one devoted to study the ASR expansion mechanisms at the level of a single matrix-aggregate interface and the other to the study of ASR expansions of cubic concrete specimens under triaxial confinement.

\subsection{Interfacial Expansion Tests}

The aim of this test is to measure the expansions at the level of a single interface aggregate-matrix due to the precipitation of ASR products. For this purpose, two kinds of cylindrical specimens of $33 \mathrm{~mm}$ diameter and $66 \mathrm{~mm}$ height are elaborated. The first type, named 'Active specimens', involves sandwich - like specimens, with cement paste or mortar on top and bottom of a disc of reactive aggregate in the middle (see Fig. 1). The second one, called 'Control specimens', involves purely mortar or cement paste specimens without reactive aggregate. Control specimens are used to assess the deformations 
of the matrix of cement paste or mortar, solely caused by phenomena such us drying shrinkage or thermal expansions. The alkali content of the cement pore solution is increased by adding $\mathrm{NaOH}$ to the mixing water.

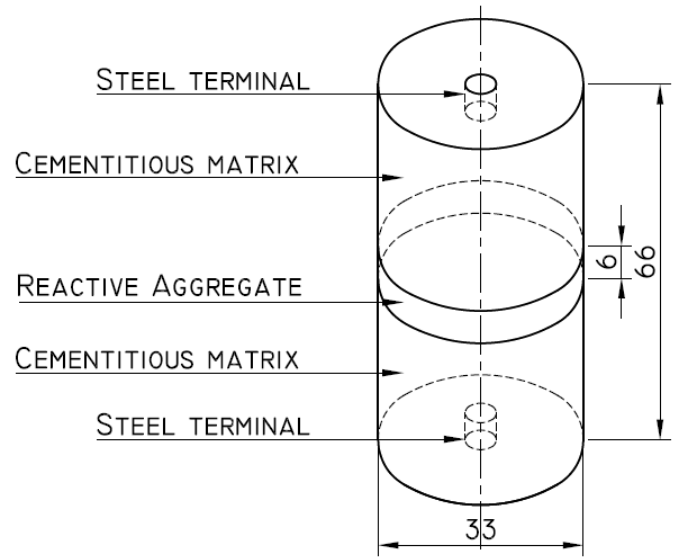

Figure 1: Configuration of active specimens used in ASR interfacial expansion tests.

So far, soda-lime glass and borosilicate glass have been used as reactive aggregates because of their advantageous of homogeneity as industrial materials. Once the methodology had been demonstrated, natural reactive aggregates will be used.

After casting, the specimens are kept in the moulds for $24 \mathrm{hs}$ in a humid chamber. Once unmoulded, they are cured in airtight containers with a solution of sodium hydroxide of $1 \mathrm{~mole} /$ litre at $23^{\circ} \mathrm{C}$ during 27 days. Finally, the containers are introduced in an oven at $60^{\circ} \mathrm{C}$ until the end of the test. The sodium hydroxide solution keeps the specimens saturated of water and prevents the leakage of alkali ions from the cement paste. During both stages, specimen length changes are measured regularly with a dial indicator until the end of the test.

It must be mentioned that a similar test has been previously proposed by Schlangen and Çopuroglu [2]. However, they have not presented, to the best of the author's knowledge, any results since then.

Once in the oven at $60^{\circ} \mathrm{C}$, the active specimens start to expand and to expel a whitish reaction product at the interfaces, until the moment when the amount of reaction product at the interface is high enough to separate the glass from the matrix, which can happen in a matter of days, weeks, or months depending on the dosage (mainly on $\mathrm{NaOH}$ added and sand content) of the matrix and the glass type.

Before the separation, the reaction products at the interface are studied by means of SEM images and EDS analysis of polished sections of the specimens. For example, in Fig. 2 a SEM image (ET detection) of the interfacial zone of an active specimen of cement paste and soda-lime glass is presented. The upper part of the image corresponds to the glass, the bottom part to the hardened cement paste (HCP) and the middle, a region of about 30 microns width, to the reaction products. Part of the epoxy used in the preparation of the sample can also be seen.

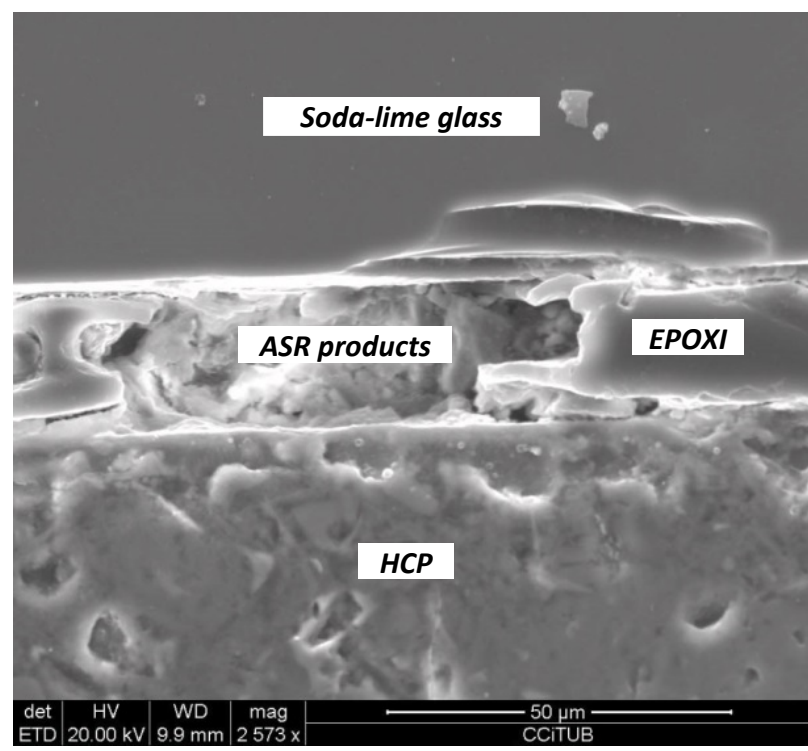

Figure 2: SEM image from the interfacial zone of an active specimen of cement paste and soda-lime glass after exposure to a $1 \mathrm{M} \mathrm{NaOH}$ solution at $60^{\circ} \mathrm{C}$.

From the length change measurements of the active and the control specimens, the expansion curves corresponding to the ASR reaction products precipitated in a single interface matrix-aggregate are obtained using Eq. (1), where $L$ and $\Delta L$ are the length and the length change of the active specimen respectively, $e$ is the width of the glass disc, $\varepsilon_{c}$ is the average deformation of the control specimens, $\alpha_{\text {glass }}$ is the thermal expansion 
coefficient of the glass, $\Delta T$ is temperature variation from curing $\left(23^{\circ} \mathrm{C}\right)$ to exposure $\left(60^{\circ} \mathrm{C}\right)$ and $d_{I}$ is the expansion at a single interface.

$d_{I}=\frac{1}{2}\left[\Delta L-\bar{\varepsilon}_{c}(L-e)-e \propto_{\text {glass }} \Delta T\right]$

A typical set of interfacial expansion curves corresponding to specimens of cement paste and soda-lime glass is presented in Fig. 3. When the curing stage is finished and the temperature is increased to $60^{\circ} \mathrm{C}$ the sudden change of the slope, from practically zero at $23^{\circ} \mathrm{C}$ to 2 microns per day, can be appreciated. After 12 days at $60^{\circ} \mathrm{C}$ the curves reached a plateau before one of the interfaces spontaneously separated. The maximum expansions measured in this case range between 25 and 35 microns per interface.

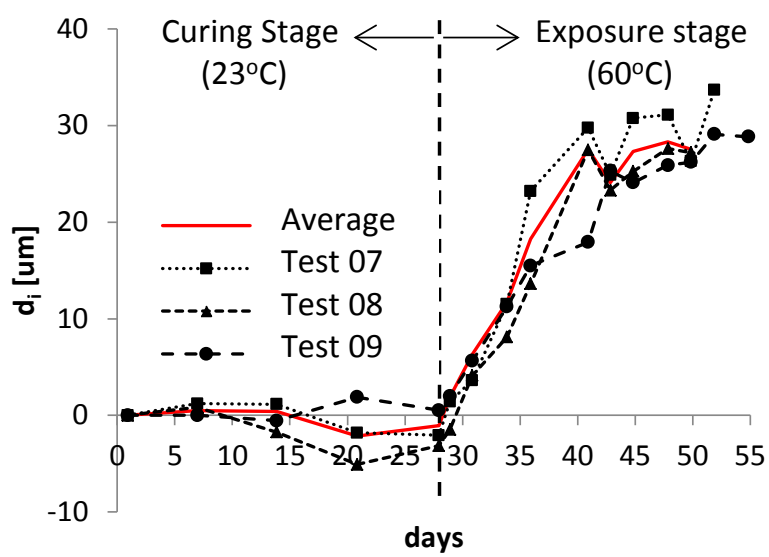

Figure 3: Interfacial expansion curves for three identical specimens of cement paste and soda-lime glass.

\subsection{Confined Expansion Tests}

The aim of this test is to measure ASR expansions in cubic concrete specimens under true triaxial confinement, i.e. under different constant stress in each main direction. To do so, an ad-hoc testing machine (AAR-TM) which was originally designed and constructed by Prof. Saouma at the U. of Colorado Boulder. Later, in 2010, it was transferred to UPC where a number of modifications were introduced to the original set-up. After several preliminary tests, the first experimental campaign with this machine is currently under way. See Fig. 4 for a general view of the equipment.

The AAR-TM allows testing cubic specimens of $150 \mathrm{~mm}$ side under different pressures from 1 to $10 \mathrm{MPa}$ in each main direction, applied by hydraulic actuators. The temperature of the specimen is controlled by means of temperature sensors and electric heaters attached to the loading plates, allowing rise the temperature up to $60^{\circ} \mathrm{C}$. The faces of the loading plates in contact with the specimens are crossed by grooves for a solution to circulate in order to keep the specimen wet and supply reactants (alkalis). Water leakage is prevented by means of an Oring in between the plate and the specimen. Additionally, the contours of the contact area are covered with silicone sealant. The dimension/shape changes of the specimen during test are measured through nine LVDTs, three in each direction, attached to the loading plates.

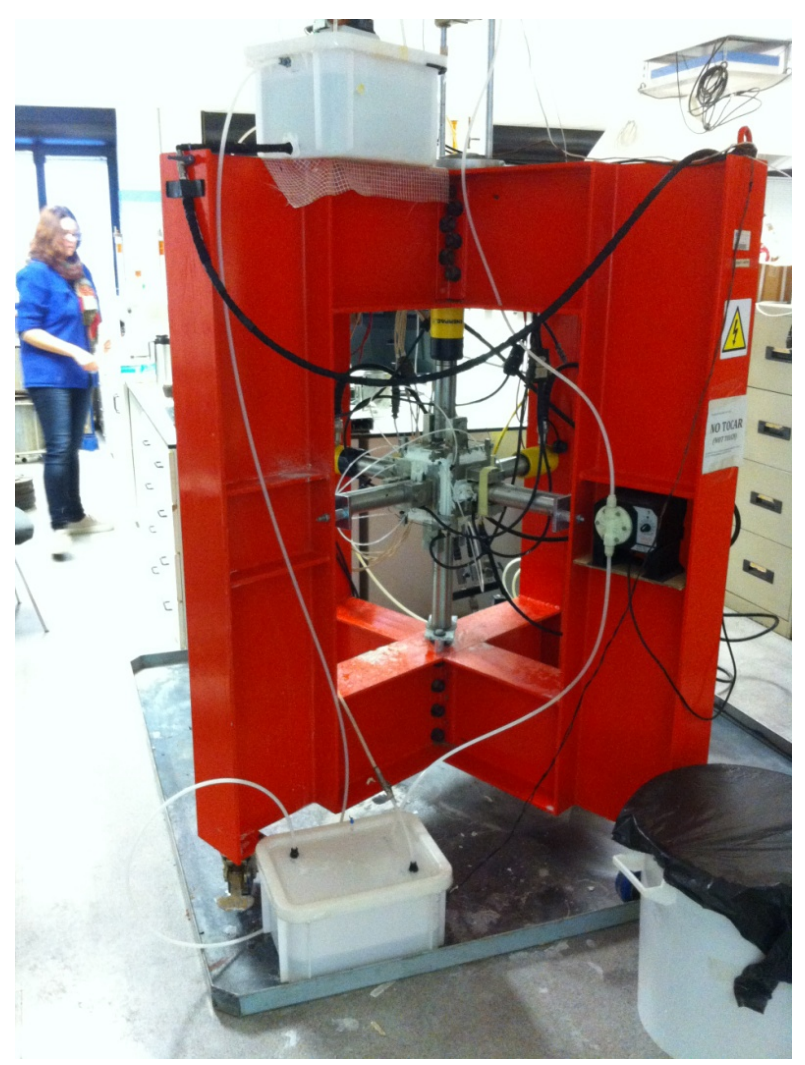

Figure 4: Machine used for ASR expansion tests under 'true' triaxial confinement states.

To isolate ASR expansions, creep deformations due to the applied load have to be deducted from the overall expansion curves 
obtained. Since, for obvious reasons, it is not possible to directly measure creep on reactive concretes, creep strains had to be assessed on concrete with similar characteristic but with non-reactive aggregates or by means of analytical expressions from the literature, always under the hypothesis that creep is not affected by ASR, i.e. the superposition principle is applicable. A similar approach is followed to deduce shrinkage strains when they are significant.

Additionally, to assess the effect of the applied confinement on the ASR expansion rate, a number of free expansion tests with specimens of identical characteristics and under the same exposure conditions (alkaline solution at $60^{\circ} \mathrm{C}$ ) were performed separately. This have to be done with a different testing procedure since the AAR-TM is not appropriated for free-expansion tests $\left(\sigma_{x}=\right.$ $\left.\sigma_{y}=\sigma_{z}=0\right)$. This procedure consists in keeping each specimen in a 19L airtight plastic recipient filled with alkaline solution inside an oven at $60^{\circ} \mathrm{C}$. The deformation of the specimen is measured regularly (once a week) in the three main directions by means of a removable $100 \mathrm{~mm}$-strain-gage which is posed on stainless-steel datum discs previously glued to the surface of the specimen.

For the first testing program (ongoing), two kinds of concrete are used: 'control concrete' and 'active concrete'. The first one is made only with non-reactive (calcareous) aggregates. The active concrete, in contrast, is made using the same mixture but replacing the coarser fraction of aggregates (sizes between $12.5 \mathrm{~mm}$ and $2.36 \mathrm{~mm}$ sieves) with crushed clear soda-lime glass.

Since only with one testing machine is available, specimens from the same batch cannot be tested simultaneously. In order to reduce the effect of concrete age in the expansion rate, specimens were tested at ages greater than 90 days. Previous free-expansion tests (not published here) had indicated significant dependence of the expansion rate on the age of the specimens for younger specimens.

In Fig. 5, preliminary results corresponding to ASR expansion curves in the three main directions of an active specimen subject to isotropic confinement $\left(\sigma_{x}=\sigma_{y}=\sigma_{z}\right)$ of 1 $\mathrm{MPa}$ are presented. For the initial register the specimen was already loaded and at testing temperature, so no elastic or thermal deformation are reflected in the expansion curves. A control specimen was tested in the same conditions showing insignificant deformations and, therefore, the curves shown in Fig. 5 need no correction by creep or shrinkage. The expansion rate obtained in the concrete casting direction (Z-direction) is significantly greater than the obtained in the other two directions. This phenomenon has been previously reported by other authors, e.g. [3], and attributed to anisotropy of the microstructure generated by the casting and compaction processes.

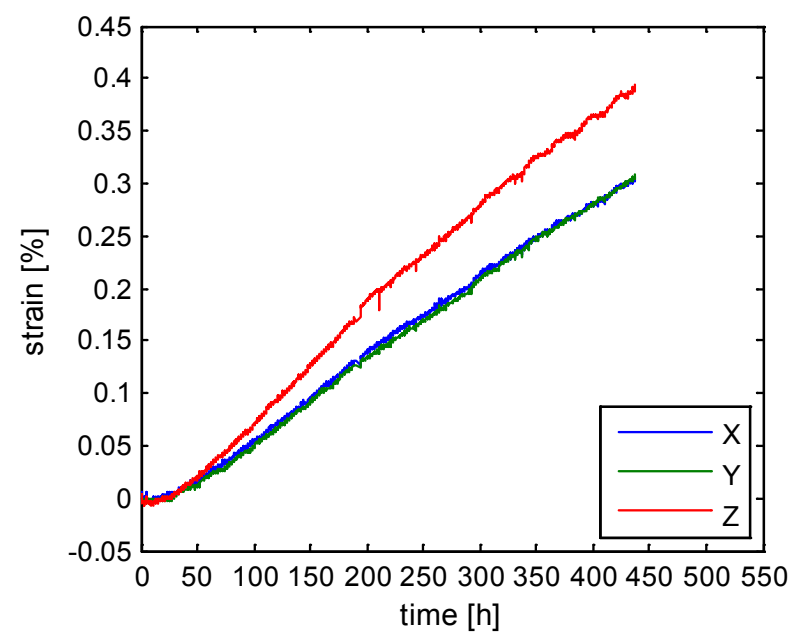

Figure 5: ASR expansion curves of cubic concrete specimens under isotropic confinement of $1 \mathrm{MPa}(\mathrm{Z}$ is the casting direction).

In Fig. 6, the average of the three expansion curves presented in Fig. 5 is contrasted with free expansion curves (also the average of the expansions in the three directions). The applied isotropic confinement seems to introduce a slight reduction of the averaged expansion rate of the material. Further testing is needed to confirm this observation. 


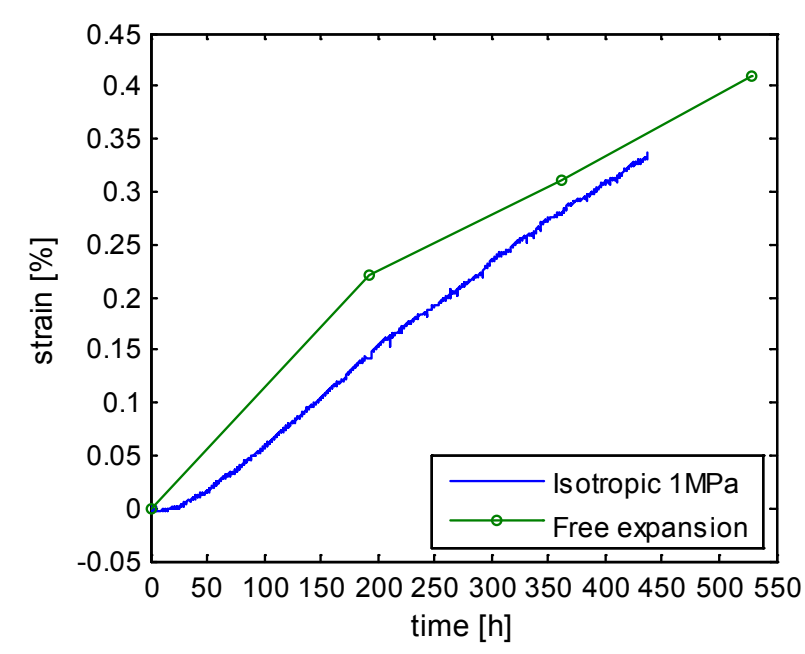

Figure 6: Comparison of isotropic confined (1MPa) expansion curve with free-expansion curves. The curves represent the average of expansions in the three directions.

\section{NUMERICAL MODELLING OF ASR}

The second part of the research project includes the formulation and numerical implementation of a chemo-mechanical model for ASR expansions in concrete at the mesolevel. In the following paragraphs, the proposed diffusion-reaction model is briefly described. This chemical formulation has been implemented in the context of a preexistent FE code (DRAC-FLOW) for the analysis of diffusion problems. As a result of ASR, local expansions over time are calculated and written in an external file that is later read by a second FE code (DRAC) for the mechanical analysis which interpret them as local pseudothermal expansions, returning the stress-strain state of the material. Previous versions of this model can be found in [4-6].

\subsection{Reaction mechanism}

The reaction mechanism proposed is schematically summarized Fig. 7. The interfacial zone between HCP and aggregate in concrete is represented. Both components are assumed to be porous and fully saturated with water at all times during the reaction. Immediately after casting, the HCP has a certain content of portlandite and its pore water has a certain content of aqueous calcium $(\mathrm{Ca})$ and alkalis $(\mathrm{R})$. Note that sodium $(\mathrm{Na})$ and potassium $(K)$ are treated here indifferently as 'alkalis' $(R)$. The aggregates are supposed to have a certain content of reactive silica $\left(\mathrm{SiO}_{2}\right)$ and no calcium or alkalis in their initial pore solution.

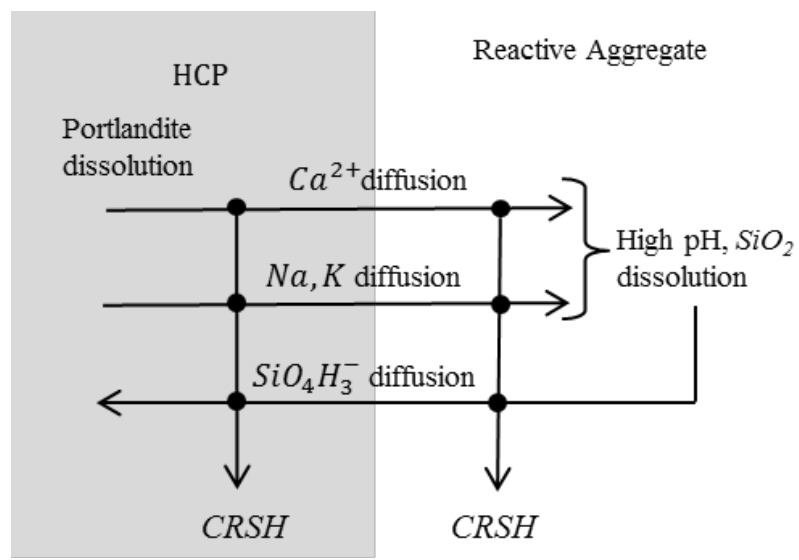

Figure 7: Schematic representation of ASR mechanism in concrete.

The concentration gradient of aqueous calcium and alkalis causes a diffusion process from the HCP towards the aggregate, which is followed by an increase of the $\mathrm{pH}$ of the pore solution in the aggregate. This increment causes the dissolution of the reactive silica into silicate ions $\left(\mathrm{SiO}_{4} \mathrm{H}_{3}^{-}\right)$, which in turn, due again to a concentration gradient, diffuse towards the cement paste. At the same time, these three diffusion processes drive the dissolution or precipitation of portlandite $\left(\mathrm{Ca}(\mathrm{OH})_{2}\right)$.

Wherever, in the aggregate or in the HCP, the three reactants (aqueous calcium, alkali and silicate) are available, a reaction occurs forming a Calcium-Alkali-Silicate-Hydrate (C$\mathrm{R}-\mathrm{S}-\mathrm{H})$ of variable composition, depending on the concentration of the reactants. The composition, in turn, determines the molar volume of this product. The effect of the remaining chemical species usually present in concrete on the development of ASR is neglected.

The variability of the composition is reached by considering two separate reactions, each one with a fixed stoichiometry and its own kinetic law, one reaction forming C-R-S$\mathrm{H}$ rich in calcium called Reaction Product $\mathrm{A}$ (RPA) and the other forming a C-R-S-H poor in calcium called Reaction Product B (RPB). 
Finally, at a given location, the volume balance of the solid constituents, namely reactive silica, portlandite, RPA and RPB, determines the transport properties and the volume of pore solution. If the volume of precipitated reaction products is greater than the available space in capillary pores plus the additional space liberated by silica and portlandite dissolution, a localized volumetric expansion may occur.

It is assumed that ASR can be suitably represented by considering the following five chemical reactions:

$$
\begin{aligned}
& \mathrm{SiO}_{2}+2 \mathrm{H}_{2} \mathrm{O} \rightleftarrows \mathrm{SiO}_{4} \mathrm{H}_{3}^{-}+\mathrm{H}^{+} \\
& \mathrm{Ca}(\mathrm{OH})_{2} \rightleftarrows \mathrm{Ca}^{2+}+2 \mathrm{OH}^{-} \\
& \mathrm{RPA} \rightleftarrows \mathrm{SiO}_{4} \mathrm{H}_{3}^{-}+a_{1} \mathrm{Ca}^{2+}+a_{2} \mathrm{R}^{+}+a_{3} \mathrm{OH}^{-} \\
& \mathrm{RPB} \rightleftarrows \mathrm{SiO}_{4} \mathrm{H}_{3}^{-}+b_{1} \mathrm{Ca}^{2+}+b_{2} \mathrm{R}^{+}+b_{4} H^{+} \\
& \mathrm{H}_{2} \mathrm{O} \rightleftarrows \mathrm{H}^{+}+\mathrm{OH}^{-}
\end{aligned}
$$

where the first four reactions are dissolution / precipitation reactions correspondent to solid silica, portlandite, RPA, and RPB, respectively, while the last one correspond to water self-ionization. $a_{i}$ and $b_{i}$ are fixed stoichiometric coefficients to be determined.

\subsection{Formulation}

Assuming that the diffusion of aqueous species in the pore solution obeys Fick's diffusion law, averaging it in the saturated porous medium and posing the correspondent mass balance equation, the following diffusion-reaction equations for the continuum porous medium are obtained

$$
\left\{\begin{array}{l}
\frac{\partial}{\partial t}\left(\phi c^{s}\right)=\nabla^{T}\left(D^{s} \nabla c^{s}\right)+q^{s} \\
\frac{\partial}{\partial t}\left(\phi c^{c}\right)=\nabla^{T}\left(D^{c} \boldsymbol{\nabla} c^{c}\right)+q^{c} \\
\frac{\partial}{\partial t}\left(\phi c^{r}\right)=\nabla^{T}\left(D^{r} \nabla c^{r}\right)+q^{r}
\end{array}\right.
$$

where the superscripts $s, c$ and $r$ indicate silicate, calcium and alkali, respectively, $\phi$ is the total porosity; $c^{\beta}\left[\mathrm{mol} / \mathrm{m}^{3}\right]$ is the concentration of aqueous $\beta$-species in the pore solution expressed in moles per unit volume of pore solution, $D^{\beta}\left[\mathrm{m}^{2} / \mathrm{s}\right]$ is the effective diffusivity of aqueous $\beta$-species in the porous medium (assumed isotropic), $\boldsymbol{\nabla}=\left[\begin{array}{ll}\frac{\partial}{\partial x} & \frac{\partial}{\partial y}\end{array}\right]^{T}$, and $q^{\beta}\left[\mathrm{mol} /\left(\mathrm{m}^{3} \cdot \mathrm{s}\right)\right]$ is the rate of production /consumption of $\beta$-species per unit volume of porous medium, which in turn is a function of the concentration of aqueous silica, calcium and alkalis, i.e. $q^{\beta}=q^{\beta}\left(c^{s}, c^{c}, c^{r}\right)$.

Analogous expressions are obtained for the localized diffusion-reaction processes occurring in a discontinuity, such as a crack or and ITZ, of width $w[\mathrm{~m}]$ and local orthogonal coordinates $(l, n)$,

$$
\left\{\begin{array}{l}
\frac{\partial}{\partial t}\left(w \phi_{m p} c_{m p}^{s}\right)=\frac{\partial}{\partial l}\left(w D_{l}^{s} \frac{\partial c_{m p}^{s}}{\partial l}\right)+q_{m p}^{s} \\
\frac{\partial}{\partial t}\left(w \phi_{m p} c_{m p}^{c}\right)=\frac{\partial}{\partial l}\left(w D_{l}^{c} \frac{\partial c_{m p}^{c}}{\partial l}\right)+q_{m p}^{c} \\
\frac{\partial}{\partial t}\left(w \phi_{m p} c_{m p}^{r}\right)=\frac{\partial}{\partial l}\left(w D_{l}^{r} \frac{\partial c_{m p}^{r}}{\partial l}\right)+q_{m p}^{r}
\end{array}\right.
$$

where the under script $m p$ indicates that the concentrations, the porosity and the sink/source rate are considered at the midplane of the discontinuity; $D_{l}^{\beta}\left[\mathrm{m}^{2} / \mathrm{s}\right]$ is the effective longitudinal diffusivity of $\beta$-species in the discontinuity. The transversal passing fluxes $j_{n}^{\beta}$ are given by

$j_{n}^{s}=D_{n}^{S} \check{c}_{m p}^{s} ; j_{n}^{c}=D_{n}^{c} \check{c}_{m p}^{c} ; j_{n}^{r}=D_{n}^{r} \breve{c}_{m p}^{r}$

where $D_{n}^{S}[\mathrm{~m} / \mathrm{s}]$ is the effective transversal diffusivity of aqueous and $\check{c}_{m p}^{s}\left[\mathrm{~mol} / \mathrm{m}^{3}\right]$ is the difference of concentration of $\beta$-species in pore solution between the two surfaces of the discontinuity. For a detailed discussion of diffusion process through discontinuities see reference [8].

A general expression of the production rate of $\beta$-species, $q^{\beta}$, is given in Eq. (10) as a function of the net rates of production of solid species resultant from Reactions (2) to (5), multiplied by the corresponding stoichiometric coefficient,

$q^{\beta}=-\sum_{\alpha} a_{\alpha}^{\beta} \Gamma^{\alpha}(\mathbb{c}, \mathcal{N})$

where $a_{\alpha}^{\beta}$ is the dimensionless stoichiometric 
coefficient of $\beta$-species in the formation reaction of solid $\alpha$-species, $\Gamma^{\alpha}\left[\mathrm{mol} /\left(\mathrm{m}^{3} \cdot \mathrm{s}\right)\right]$ is the reaction rate of solid $\alpha$-species per unit volume of porous material (positive for solid formation, negative for solid dissolution), which is a function of the vector of local concentration of aqueous species $\mathbb{C}=$ $\left[\begin{array}{lll}c^{s} & c^{c} & c^{r}\end{array}\right]$, expressed in moles per unit volume of pore solution and of the vector of local content of solid species $\mathcal{N}=$ $\left[\begin{array}{llll}\mathcal{N}^{S} & \mathcal{N}^{C} & \mathcal{N}^{R P A} & \mathcal{N}^{R P B}\end{array}\right]$ which is expressed in moles per unit volume of porous material.

The calculation of the sink/source terms $q^{\beta}$ according to Eq. (10) requires stablishing the kinetic laws for Reactions (2) to (5) in order to obtained the correspondent reaction rates $\Gamma^{\alpha}$. To do so, it is assumed that the driving force of the dissolution / precipitation reaction of reactive solid $\alpha$-species is $\left(\psi^{\alpha}-1\right)$, where $\psi^{\alpha}$ is the saturation index of the pore solution with respect to solid $\alpha$-species. Dissolution and precipitation reactions are assumed not to occur simultaneously but alternately depending on $\psi^{\alpha}$. If $\psi^{\alpha}>1$ the solution is over-saturated with respect to $\alpha$-species and, consequently, the reaction progresses in the precipitation direction. If $\psi^{\alpha}<1$, the solution is under-saturated and solid dissolution occurs. If $\psi^{\alpha}=1$, the solid and the solution are in thermodynamical equilibrium. The saturation indexes considered for Reactions (2) to (5) are

$\psi^{S}=\frac{a^{s} a^{h}}{K_{s p}^{S}}$

$\psi^{C}=\frac{a^{c}\left(a^{o h}\right)^{2}}{K_{s p}^{C}}$

$\psi^{R P A}=\frac{a^{S}\left(a^{c}\right)^{a_{1}}\left(a^{r 1}\right)^{a_{2}}\left(a^{o h}\right)^{a_{3}}}{K_{s p}^{R P A}}$

$\psi^{R P B}=\frac{a^{S}\left(a^{c}\right)^{b_{1}}\left(a^{r 1}\right)^{b_{2}}\left(a^{h}\right)^{a_{4}}}{K_{s p}^{R P B}}$

where $a^{\beta}$ is the thermodynamic activity of aqueous $\beta$-species in pore solution, $K_{s p}^{\alpha}$ is the saturation product constant of $\alpha$-species dissolution, and $a_{i}, b_{i}$ are the stoichiometric coefficients of RPA and RPB reactions.

The thermodynamical activity of aqueous $\beta$-species in solution is calculated as

$a^{\beta}=\gamma^{\beta} c^{\beta}$

where $\gamma^{\beta}$ is the activity coefficient which is calculated with Davies Equation.

In order to determine the activities of the chemical species intervening in the kinetics laws described above, it is essential to determine the equilibrium concentrations of $\mathrm{H}^{+}$and $\mathrm{OH}^{-}$for given concentrations of silicates $\left(c^{s}\right)$, calcium $\left(c^{c}\right)$ and alkalis $\left(c^{r}\right)$ in the solution. This is achieved by solving a non-linear system of equations given by the equilibrium equation of Reaction (6) as well as the electric charge balance equation of the pore solution.

The mass balance equation of solid $\alpha$ species is given by

$\frac{\partial\left(\mathcal{N}^{\alpha} \mathcal{U}\right)}{\partial t}=\mathcal{U} \Gamma^{\alpha}$

where $\mathcal{N}^{\alpha} \mathcal{U}$ is the total quantity of (the total number of moles of) solid $\alpha$-species.

The total volume of porous medium or discontinuity, $\mathcal{U}\left[\mathrm{m}^{3}\right]$, is given by

$u=u^{c p}+\sum_{\alpha} u^{\alpha}+\sum_{\vartheta} u^{\vartheta}$

where $\mathcal{U}^{\alpha}$ is the volume of reactive solid $\alpha$ species, $\mathcal{U}^{\vartheta}$ is the volume of inert solid phase $\mathcal{U}^{\vartheta}$, and $\mathcal{U}^{c p}$ is the part of the material volume not occupied by the solid phases and which is assumed to be filled with free water.

The volume of reactive solid $\alpha$-species is obtained as function of their molar concentration with

$\mathcal{U}^{\alpha}=\left(\frac{\eta^{\alpha}}{1-\phi^{\alpha}}\right) \mathcal{N}^{\alpha} u$

where $\eta^{\alpha}$ is the molar volume and $\phi^{\alpha}$ is the intrinsic porosity of solid $\alpha$-species. Intrinsic porosity is defined as the ratio between gel water volume and total apparent volume of phase $\alpha$. Note that for silica and portlandite the intrinsic porosity is null $\left(\phi^{S}=\phi^{C}=0\right)$.

The $\mathcal{U}^{c p}$ in discontinuities and the continuous porous medium are calculated with 
$u^{c p}=\left\langle 1-\sum_{\alpha} u^{\alpha}-\sum_{\vartheta} \mathcal{u}^{\vartheta}\right\rangle$

where the Macaulay brackets $\langle X\rangle$ stands for 'the positive part of $X$ ', i.e. if $X<0,\langle X\rangle=0$ otherwise $\langle X\rangle=X$.

Since concrete is assumed to be fully saturated with water, the total volume of pore solution $\mathcal{U}^{p s}$, which includes free and gel water, is equal to the total volume of pores and clean crack aperture, i.e.

$\mathcal{U}^{p s}=\mathcal{U}^{c p}+\sum_{\alpha} \phi^{\alpha} \mathcal{U}^{\alpha}+\sum_{\vartheta} \phi^{\vartheta} \mathcal{U}^{\vartheta}$

Finally, the total porosity $\phi$ is given by

$\phi=\frac{u^{p s}}{u}$

Eventually, the volume of Reaction Products (RPs) may grow to a point in which the available space is exhausted, i.e. $\mathcal{U}^{c p}=0$. From this point on, any additional volume of RPs will impose strains (in the continuous porous medium) or displacements (if ASR is occurring in a discontinuity) at the reaction site. The magnitude of these imposed strains or displacements is obtained under the hypothesis that the RPs are incompressible and that their apparent molar volumes are independent of their tensional state. These assumptions lead to the following expressions of the ASR-imposed volumetric strain $\left(\varepsilon_{v}^{o}\right)$ in the continuous porous medium

$\frac{\partial \varepsilon_{v}^{o}}{\partial t}=\left\{\begin{array}{cl}0 & \text { if } \mathcal{U}^{c p}>0 \\ \frac{\partial \mathcal{U}}{\partial t} & \text { if } \mathcal{U}^{c p}=0\end{array}\right.$

and the ASR-imposed normal aperture $a_{n}^{o}$ in discontinuities

$\frac{\partial a_{n}^{o}}{\partial t}=\left\{\begin{array}{cl}0 & \text { if } \mathcal{U}^{c p}>0 \\ \frac{\partial \mathcal{U}}{\partial t}-\frac{\partial a_{n}^{e p}}{\partial t} & \text { if } \mathcal{U}^{c p}=0\end{array}\right.$

where $\partial a_{n}^{e p} / \partial t$ stands for the aperture rate due to mechanical loads. Positive $\partial a_{n}^{e p} / \partial t$ increases the space available to accommodate reaction products reducing the rate of chemical imposed aperture. Note that in the continuum medium the rate of volumetric deformation due to mechanic loads has been neglected, since it is expected that $\partial \varepsilon_{v}^{e p} / \partial t \ll \partial \mathcal{U} / \partial t$.

\subsection{Numerical implementation}

The model has been implemented by means of Finite Elements Method in a way similar to other chemical attacks previously developed in our research group (MECMAT-UPC) [7].

\subsection{Preliminary results}

The simplicity of the geometry of the interfacial expansion tests described above make them especially suitable for numerical simulations with the purpose of validating or calibrating the proposed ASR model. In particular, a set of tests performed with specimens provided with a complete lateral sealing in order to prevent mass exchange (described in detail in Ref. [6]) can be adequately simulated by means $1 \mathrm{D}$ models. In this section, preliminary simulation results of these tests obtained with the proposed ASR model are presented.

The active specimens considered were made with soda-lime glass and cement paste and tested according to the procedure described above. In Fig. 7, the obtained interfacial expansion curves of four specimens during the exposure stage are presented.

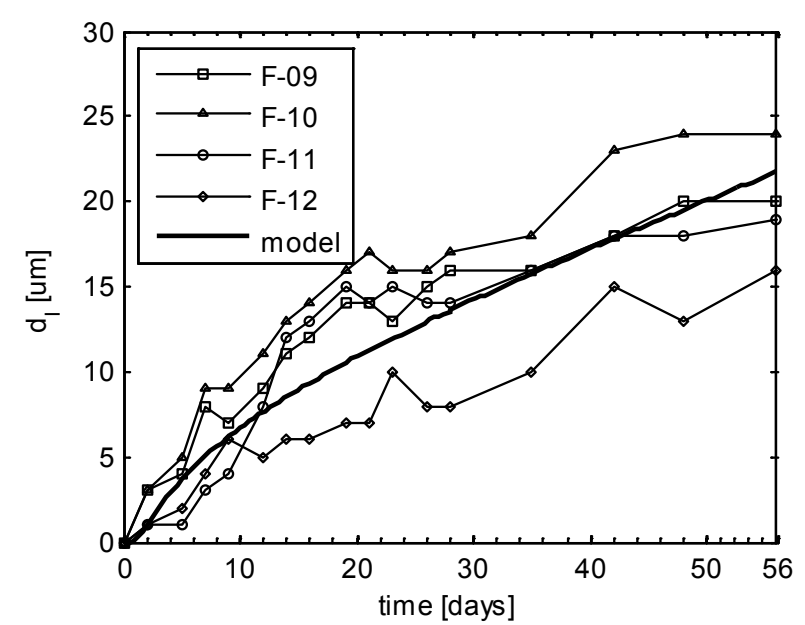

Figure 7: Interfacial expansion curves at exposure stage of glass-cement paste specimens with complete lateral sealing. Experimental and modelling results.

The kinetics law considered for portlandite, RPA and RPB reactions are of the form 
$\Gamma^{\alpha}=\left\{\begin{array}{l}\phi k_{d}^{\alpha}\left(\psi^{\alpha}-1\right) \text { if } \psi^{\alpha}<1 ; \mathcal{N}^{\alpha}>0 \\ \phi k_{f}^{\alpha}\left(\psi^{\alpha}-1\right) \text { if } \psi^{\alpha}>1\end{array}\right.$

For silica reactions, in contrast, the porosity $\phi$ has been removed of the kinetic law, i.e.

$\Gamma^{S}=\left\{\begin{array}{l}k_{d}^{S}\left(\psi^{S}-1\right) \text { if } \psi^{S}<1 ; \mathcal{N}^{S}>0 \\ k_{f}^{S}\left(\psi^{S}-1\right) \text { if } \psi^{S}>1\end{array}\right.$

The geometry of the model is schematically represented in Fig. 8. The FE mesh consists of a 'column' of 149 quadrangular elements (42 for glass and 107 for HCP) separated with 1 joint element representing the ITZ; a total of 302 nodes. The size of the continuum elements was reduced near to the ITZ and at the top of the specimen, where the highest concentration gradients were expected.

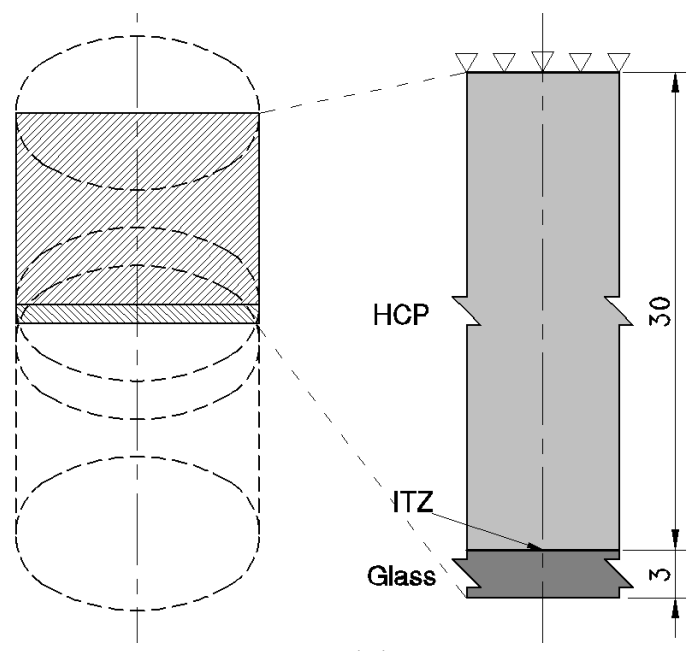

Figure 8: Model geometry.

At the upper boundary, constant concentrations of the three diffusive species were imposed $\left(c^{s}=c^{c}=0, c^{r}=1000\right)$. The remaining boundaries were considered impervious. Initial concentrations at $\mathrm{HCP}$ were assumed $c^{s}=0, c^{c}=0.03$ and,$c^{r}=$ 700 and the initial concentrations in the glass were assumed as $c^{s}=c^{c}=c^{r}=0$. All concentrations are expressed in $\mathrm{mol} / \mathrm{m}^{3}$.

The volumetric fractions of solid constituents of the HCP, ITZ and aggregate (glass) considered in the model are summarized in Table 1, while the chemical parameters of solid species are summarized in Table 2. Additionally, $\phi^{I C P}=0.165$ was assumed. Note that glass was considered to be formed exclusively by silica. The effect of the other chemical components of the glass, if any, were not considered. The adopted stoichiometric coefficients correspondent to Reactions (4) and (5) were $a_{1}=1.5, a_{2}=0.2$, $a_{3}=2.2, b_{1}=0.3, b_{2}=0.3$ and $b_{4}=0.1$.

Table 1: Initial volumetric fractions.

\begin{tabular}{cccc}
\hline & HCP & ITZ & Glass \\
\hline $\mathcal{U}^{S}$ & $0.00 \mathrm{E}+00$ & $0.00 \mathrm{E}+00$ & $1.00 \mathrm{E}+00$ \\
$\mathcal{U}^{c}$ & $0.20 \mathrm{E}+00$ & $0.00 \mathrm{E}+00$ & $0.00 \mathrm{E}+00$ \\
$\mathcal{U}^{I C P}$ & $0.60 \mathrm{E}+00$ & $0.00 \mathrm{E}+00$ & $0.00 \mathrm{E}+00$ \\
$\mathcal{U}^{I A G}$ & $0.00 \mathrm{E}+00$ & $0.00 \mathrm{E}+00$ & $0.00 \mathrm{E}+00$ \\
$\mathcal{U}^{I R}$ & $0.00 \mathrm{E}+00$ & $0.00 \mathrm{E}+00$ & $0.00 \mathrm{E}+00$ \\
$\mathcal{U}^{c p}$ & $0.20 \mathrm{E}+00$ & $0.00 \mathrm{E}+00$ & $0.00 \mathrm{E}+00$ \\
$\mathcal{U}$ & $1.00 \mathrm{E}+00$ & $1.00 \mathrm{E}-07$ & $1.00 \mathrm{E}+00$ \\
\hline
\end{tabular}

Table 2: Chemical parameters of solid species.

\begin{tabular}{ccccc}
\hline$\alpha$ & $\mathrm{S}$ & $\mathrm{C}$ & $\mathrm{RPA}$ & $\mathrm{RPB}$ \\
\hline$\eta^{\alpha}$ & $2.22 \mathrm{E}-05$ & $3.32 \mathrm{E}-05$ & $6.72 \mathrm{E}-05$ & $4.60 \mathrm{E}-05$ \\
$\phi^{\alpha}$ & $1.00 \mathrm{E}-03$ & $0.00 \mathrm{E}+00$ & $0.40 \mathrm{E}+00$ & $0.74 \mathrm{E}+00$ \\
$K_{s p}^{\alpha}$ & $4.01 \mathrm{E}-06$ & $6.83 \mathrm{E}+03$ & $5.00 \mathrm{E}+02$ & $1.50 \mathrm{E}+00$ \\
$k_{d}^{\alpha}$ & $2.00 \mathrm{E}+00$ & $5.00 \mathrm{E}+02$ & $4.00 \mathrm{E}+01$ & $5.00 \mathrm{E}+01$ \\
$k_{f}^{\alpha}$ & $5.00 \mathrm{E}+00$ & $1.00 \mathrm{E}-02$ & $1.00 \mathrm{E}+01$ & $1.00 \mathrm{E}+00$ \\
\hline
\end{tabular}

By means of SEM images, it has been determined that the formation of RP mainly occurs in the interface between HCP and glass (see Fig. 2). This fact allowed us to further simplify of the model by restricting the reactions corresponding to silica and portlandite dissolution/precipitation to occur in the HCP and glass domains, while the reactions corresponding to the RPA and RPB were restricted to the ITZ.

The effective diffusivities through the three domains considered were assumed constant in accordance with Table 3.

Table 3: Effective difusivities for the continuous and interface elements expressed in $\mathrm{m}^{2} / \mathrm{h}$ and $\mathrm{m} / \mathrm{h}$, respectively.

\begin{tabular}{lccc}
\hline & $D^{s}$ & $D^{c}$ & $D^{r}$ \\
\hline HCP & $3.65 \mathrm{E}-08$ & $2.46 \mathrm{E}-08$ & $4.16 \mathrm{E}-08$ \\
Glass & $9.11 \mathrm{E}-11$ & $6.12 \mathrm{E}-11$ & $1.04 \mathrm{E}-11$ \\
\hline & $D_{n}^{s}$ & $D_{n}^{c}$ & $D_{n}^{r}$ \\
\hline ITZ & $1.00 \mathrm{E}+00$ & $1.00 \mathrm{E}+00$ & $1.00 \mathrm{E}+00$ \\
\hline
\end{tabular}

The expansion curve obtained with the model is plotted in Fig. 7, together with the 
corresponding experimental ones. Both the final expansion and the kinetics of the process seem to be adequately reproduced by the model.

In order to illustrate the reaction mechanism, the time evolution of the volumetric fractions at representative points of the three domains considered is illustrated in Fig. 9. As the ASR develops, portlandite (Fig. 9a) and glass (Fig. 9c) dissolve with the consequent increment of capillary porosity. The resulting calcium and silicate ions diffuse through the ITZ, driving the formation of RPA and RPB (Fig. 9b).

The averaged composition of the reaction product in the ITZ the end of the test has calcium to silica and alkali to silica molar ratios of $\mathrm{Ca} / \mathrm{Si}=1.41$ and $\mathrm{R} / \mathrm{Si}=0.04$. These ratios are in good agreement with values measured on similar specimens tested in former interfacial tests $(\mathrm{Ca} / \mathrm{Si}=1.1$ to 1.3 , $\mathrm{R} / \mathrm{Si}=0.05$ to 0.30 ).

\section{CONCLUDING REMARKS}

An ongoing research project on the mechanisms of concrete deterioration due to Alkali-Silica Reaction (ASR) has been presented, together with some preliminary results. The research program includes both experimental work and numerical modelling.

The first part includes the development of two new experimental setups, one devoted to study the ASR expansion mechanisms at the level of a single matrix-aggregate interface and the other to the study of ASR expansions of cubic concrete specimens under triaxial confinement.

The second part includes the formulation and numerical implementation of a chemomechanical model for ASR expansions in concrete at the meso-level.

Preliminary results of the simulation of interfacial expansion test indicate that the proposed is capable of reproducing the kinetics of ASR expansions as well as the chemical composition of the reaction products.
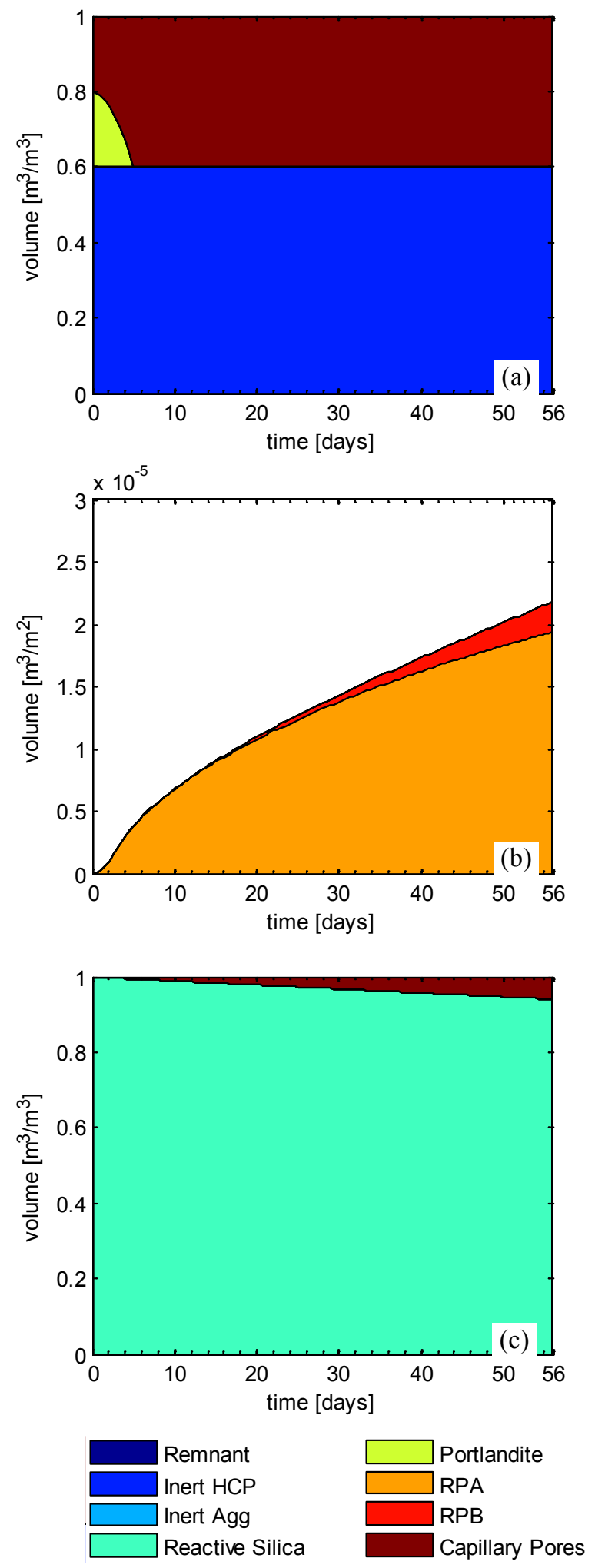

Figure 9: Time evolution of volumetric fractions for integration points at (a) HCP, (b) ITZ and (c) glass.

\section{ACKNOWLEDGMENTS}

This research is supported by grants BIA2012-36898 funded by MEC (Madrid), which includes FEDER funds, and 2014SGR-1523 
from AGAUR-Generalitat de Catalunya (Barcelona).

\section{REFERENCES}

[1] Stanton, T. E. 1940. Expansion of concrete through reaction between cement and aggregate. Proceedings of American Society of Civil Engineers 66: 1781-1811.

[2] Schlangen, E., \& Çopuroglu, O. 2007. Concrete damage due to alkali-silica reaction: A new method to determine the properties of expansive gel. In Carpinteri et al. (eds.), Fracture Mechanics of Concrete and Concrete Structures - HighPerformance Concrete, Brick-Masonry and Environmental Aspects; London: Taylor \& Francis Group; pp. 1835-1841.

[3] Multon, S., Seignol, J., and Toutlemonde, F. 2005. Structural Behavior of Concrete Beams Affected by Alkali - Silica Reaction. ACI Materials Journal, 102(2): 67-76.

[4] Liaudat, J., López, C., and Carol, I. 2013. Diffusion-reaction model for alkali-silica reaction in concrete. In Oñate, E. et al. (eds.) Computational Plasticity XII Fundamentals and Applications; Barcelona: International Center for Numerical Methods in Engineering (CIMNE); pp. 479-489.

[5] Liaudat, J., López, C., and Carol, I. 2014. Diffusion-reaction model for ASR : formulation and 1D numerical implementation. In Bicanic, N. et al. (eds.) Computational Modelling of Concrete and Concrete Structures; CRC Press; v.2, pp. 639-648.

[6] Liaudat, J., Martínez, A., López, C., and Carol, I. 2015. Numerical Modelling of ASR Expansions in Concrete. In Hellmich, C. et al. (eds.) CONCREEP 10: Mechanics and Physics of Creep, Shrinkage, and Durability of Concrete and Concrete Structures; ASCE, pp. 445454.

[7] Idiart, A., López, C., and Carol, I. 2011. Chemo-mechanical analysis of concrete cracking and degradation due to external sulfate attack: A meso-scale model.
Cement and Concrete Composites, 33(3): 411-423.

[8] Segura, J. M. \& Carol, I., 2004. On zerothickness interface elements for diffusion problems. International Journal for Numerical and Analytical Methods in Geomechanics, 28(9), 947-962. 